Vietnam Journal of Mechanics, VAST, Vol.34, No. 2 (2012), pp. $67-77$

\title{
THREE-POINT CORRELATION BOUNDS ON THE EFFECTIVE BULK MODULUS OF ISOTROPIC MULTICOMPONENT MATERIALS
}

\author{
Pham Duc Chinh, Vu Lam Dong \\ Institute of Mechanics, VAST
}

\begin{abstract}
Three-point correlation bounds based on minimum energy principles are constructed to give estimates on the effective elastic bulk modulus of disordered multicomponent materials. The constructed trial fields are extensions of Hashin-Shtrikman polarization ones used in our previous approach and lead to tighter bounds. Some examples of applications are presented.
\end{abstract}

Key words: Isotropic multicomponent material, effective elastic moduli, three-point correlation parameters.

\section{INTRODUCTION}

Determination of effective elastic moduli of composite materials, especially those with disordered irregular micro-geometries, is a difficult task, and in many cases one has to construct upper and lower bounds on the moduli, using limited available statistical information about the micro-structure of the composites. That can be done with the help of variational approach [1 - 6]. The most simple bounds are Voigt-Reuss-Hill arithmetic average and harmonic average ones based on minimum energy and complementary energy principles, with constant trial stress and strain fields. Hashin-Shtrikman [1] constructed tight bounds that also involve only the information on the volume proportions and components properties of the isotropic composites, developing their special variational principles and ingenious polarization trial fields. Starting from the classical minimum energy principles and using Hashin-Shtrikman polarization trail fields, Pham [2] obtained the bounds tighter than Hashin-Shtrikman ones that involve additional three-point correlation parameters describing the micro-structure of a composite.

In this work, we use new trial field more general than Hashin-Shtrikman polarization ones to derive three-point bounds on the effective elastic bulk modulus tighter than those constructed in [2]. Applications of the bounds are given to some composites, three-point correlation information about which is available in literature.

\section{CONSTRUCTION OF BOUNDS}

The effective elastic moduli $\mathbf{C}^{e f f}=\mathbf{T}\left(k^{e f f}, \mu^{e f f}\right)$ of an isotropic $n$-component material can be defined via the minimum energy expression on the representative volume 
element $V[1,6]$

$$
\varepsilon^{0}: \mathbf{C}^{e f f}: \varepsilon^{0}=\inf _{\langle\varepsilon\rangle=\varepsilon^{0}} \int_{V} \varepsilon: \mathbf{C}: \varepsilon d \mathbf{x}
$$

with the component elastic moduli $\mathbf{C}(\mathbf{x})=\mathbf{T}\left(k_{\alpha}, \mu_{\alpha}\right)$ if $\mathbf{x} \in V_{\alpha} \subset V, \alpha=1, \ldots, n$; where $\mathbf{T}$ is the isotropic fourth-rank tenser with components

$$
T_{i j k l}(k, \mu)=k \delta_{i j} \delta_{k l}+\mu\left(\delta_{i k} \delta_{j l}+\delta_{i l} \delta_{j k}-\frac{2}{3} \delta_{i j} \delta_{k l}\right),
$$

$\delta_{i j}$ is Kronecker delta, the compatible strain field is expressible via the displacement field $\mathbf{u}(\mathbf{x})$

$$
\varepsilon(\mathbf{x})=\frac{1}{2}\left[\nabla \mathbf{u}+(\nabla \mathbf{u})^{T}\right] ; \quad \mathbf{x} \in V
$$

〈. $\rangle$ means the volume average on $\mathrm{V}, \varepsilon^{0}$ is a constant strain field.

To construct the best possible upper bound on the effective bulk modulus $k^{\text {eff } f}$ from (1), we choose the following compatible strain trial field

$$
\varepsilon_{i j}=\left(\frac{\delta_{i j}}{3}+\sum_{\alpha=1}^{n} a_{\alpha} \varphi_{, i j}^{\alpha}\right) \varepsilon^{0} ; \quad i, j=1,2,3,
$$

where $\varepsilon_{i j}^{0}=\frac{\delta_{i j}}{3} \varepsilon^{0}$ is a constant volumetric strain, $\varphi^{\alpha}$ is the harmonic potential, Latin indices after comma designate differentiation with respective Cartesian coordinates.

$$
\varphi^{\alpha}(\mathbf{x})=-\int_{V_{\alpha}} \frac{1}{4 \pi|\mathbf{x}-\mathbf{y}|} d \mathbf{y} ; \quad \nabla^{2} \varphi^{\alpha}(\mathbf{x})=\delta_{\alpha \beta}, \quad \mathbf{x} \in V_{\beta},
$$

$a_{\alpha}$ are free scalar that satisfy the restrictions [for the trial field to satisfy the restriction $\langle\varepsilon\rangle=\varepsilon^{0}$ in $\left.(1)\right]$

$$
\sum_{\alpha=1}^{n} v_{\alpha} a_{\alpha}=0
$$

$v_{\alpha}$ is volume fraction of $\alpha$-phase. The volume of $\mathrm{V}$ is assumed to be unity.

Three-point correlation parameters $A_{\alpha}^{\beta \gamma}$ describing the micro-structure of a composite are defined as $(\alpha, \beta, \gamma=1, \ldots, n)[2]$

$$
A_{\alpha}^{\beta \gamma}=\int_{V_{\alpha}} \varphi_{i j}^{\beta \alpha} \varphi_{i j}^{\gamma \alpha} d \mathbf{x}, \quad \varphi_{i j}^{\beta \alpha}=\varphi_{, i j}^{\beta}-\frac{1}{v_{\alpha}} \int_{V_{\alpha}} \varphi_{, i j}^{\beta} d \mathbf{x}
$$

conventional summation on the repeating Latin indices is assumed. We have relations [7]:

$$
\sum_{\beta=1}^{n} A_{\alpha}^{\beta \gamma}=0, \quad \forall \alpha, \gamma=1, \ldots, n .
$$

The three-point correlation parameters $A_{\alpha}^{\beta \gamma}$ relate the microgeometies of three components $V_{\alpha}, V_{\beta}, V_{\gamma}$. In principle, one could characterize fully the microgeometry of a composite with $n$-point correlation parameters, up to $n \rightarrow \infty$. One-point correlation parameters are just 
the volume fractions of the components. Two-point correlation parameters do not appear in the expressions of the effective moduli of macroscopically isotropic composites (but in those for anisotropic composites). Numerical determinations of the multipoint correlation parameters for particular microstructures are difficult tasks. At present only the values of the three-point ones, and for just two-component materials, are tabulated for a number of microgeometries in the literature.

Substituting the trial field (4) into energy functional (1), one obtains:

$$
W_{\boldsymbol{\varepsilon}}=\int_{V} \boldsymbol{\varepsilon}: \mathbf{C}: \boldsymbol{\varepsilon} d \mathbf{x}=\left[k_{V}+\sum_{\alpha=1}^{n} v_{\alpha} k_{\alpha}\left(2 a_{\alpha}+a_{\alpha}^{2}\right)+\sum_{\alpha, \beta, \gamma=1}^{n} A_{\alpha}^{\beta \gamma} 2 \mu_{\alpha} a_{\beta} a_{\gamma}\right]\left(\varepsilon^{0}\right)^{2},
$$

where $k_{V}$ is Voigt arithmetic average

$$
k_{V}=\sum_{\alpha=1}^{n} v_{\alpha} k_{\alpha}
$$

We minimize (9) over the free variables $a_{\alpha}$ restricted by (6) with the help of Lagrange multiplier $\lambda$ and get the equations

$$
v_{\alpha} k_{\alpha}+v_{\alpha} k_{\alpha} a_{\alpha}+\sum_{\beta, \gamma=1}^{n} A_{\gamma}^{\alpha \beta} 2 \mu_{\gamma} a_{\beta}-\lambda v_{\alpha}=0, \quad \alpha=1, \ldots, n
$$

Equation (11) can be presented in an absolute notation form

$$
\boldsymbol{A}_{k} \cdot \mathbf{a}+\mathbf{v}_{k}-\lambda \mathbf{v}=\mathbf{0}
$$

which has the formal solution

$$
\mathbf{a}=\mathcal{A}_{k}^{-1} \cdot\left(\lambda \mathbf{v}-\mathbf{v}_{k}\right),
$$

where we have introduced the vectors $\mathbf{a}, \mathbf{v}, \mathbf{v}_{k}$ and matrix $\mathcal{A}_{k}$ in n-space

$$
\begin{aligned}
& \mathbf{a}=\left\{a_{\alpha}\right\}_{\alpha}^{n}, \quad \mathbf{v}=\left\{v_{\alpha}\right\}_{\alpha}^{n}, \quad \mathbf{v}_{k}=\left\{v_{\alpha} k_{\alpha}\right\}_{\alpha}^{n}, \\
& \mathcal{A}_{k}=\left\{v_{\alpha} k_{\alpha} \delta_{\alpha \beta}+\sum_{\gamma=1}^{n} A_{\gamma}^{\alpha \beta} 2 \mu_{\gamma}\right\}_{\alpha \beta}^{n} .
\end{aligned}
$$

With (13), eq. (6) can be presented as

$$
0=\mathbf{v} \cdot \mathbf{a}=\mathbf{v} \cdot \mathcal{A}_{k}^{-1} \cdot\left(\lambda \mathbf{v}-\mathbf{v}_{k}\right),
$$

which is solved over $\lambda$

$$
\lambda=\frac{\mathbf{v} \cdot \mathcal{A}_{k}^{-1} \cdot \mathbf{v}_{k}}{\mathbf{v} \cdot \mathcal{A}_{k}^{-1} \cdot \mathbf{v}}
$$

Now taking sum of eqs. (11) on $\alpha$ from 1 to $\mathrm{n}$ and keeping in mind eq. (8) and eq. (10), one derives

$$
k_{V}+\sum_{\alpha=1}^{n} v_{\alpha} k_{\alpha} a_{\alpha}-\lambda=0
$$


Using (11),(6), we simplify (9) to

$$
W_{\epsilon}=\left(k_{V}+\sum_{\alpha=1}^{n} v_{\alpha} k_{\alpha} a_{\alpha}\right)\left(\varepsilon^{0}\right)^{2}=\lambda\left(\varepsilon^{0}\right)^{2} .
$$
from (1)

Taking in account (17) with (9), (16), (18), one obtains the upper bound on $k^{\text {eff }}$

$$
k^{e f f} \leq K_{A}^{U}\left(\left\{k_{\alpha}\right\}_{\alpha}^{n},\left\{\mu_{\alpha}\right\}_{\alpha}^{n},\left\{v_{\alpha}\right\}_{\alpha}^{n},\left\{A_{\alpha}^{\beta \gamma}\right\}_{\alpha \beta \gamma}^{n}\right)=\frac{\mathbf{v} \cdot \mathcal{A}_{k}^{-1} \cdot \mathbf{v}_{k}}{\mathbf{v} \cdot \mathcal{A}_{k}^{-1} \cdot \mathbf{v}}
$$

To construct the lower bound on the effective moduli we use the minimum complementary energy principle

$$
\boldsymbol{\sigma}^{0}:\left(\mathbf{C}^{e f f}\right)^{-1}: \boldsymbol{\sigma}^{0}=\inf _{\langle\boldsymbol{\sigma}\rangle=\boldsymbol{\sigma}^{0}} \int_{V} \boldsymbol{\sigma}:(\mathbf{C})^{-1}: \boldsymbol{\sigma} d \mathbf{x},
$$

where $\boldsymbol{\sigma}^{0}$ is a constant stress field, and the stress field $\boldsymbol{\sigma}$ should satisfy equilibrium equation

$$
\nabla \cdot \boldsymbol{\sigma}(\mathbf{x})=\mathbf{0}, \quad \mathbf{x} \in V .
$$

To find the best possible lower bound on $k^{\text {eff }}$ from (20) we take the following equilibrated stress trial field

$$
\sigma_{i j}=\left[\delta_{i j}+\sum_{\alpha=1}^{n} a_{\alpha}\left(\varphi_{, i j}^{\alpha}-\delta_{i j} \mathcal{I}^{\alpha}\right)\right] \sigma^{0} ; \quad i, j=1,2,3,
$$

where $\sigma_{i j}^{0}=\delta_{i j} \sigma^{0}$ is a constant hydrostatic field, $\mathcal{I}^{\alpha}$ is an indicator function

$$
\mathcal{I}^{\alpha}(\mathbf{x})= \begin{cases}1, & \mathbf{x} \in V_{\alpha} \\ 0, & \mathbf{x} \notin V_{\alpha}\end{cases}
$$

$a_{\alpha}$ are free scalars subjected to the same restriction (6).

Substituting the trial field (22) into (20) and following procedure similar to that form (9) to (19), one obtaines the best possible lower bound on $k^{\text {eff }}$

$$
k^{e f f} \geq K_{A}^{L}\left(\left\{k_{\alpha}\right\}_{\alpha}^{n},\left\{\mu_{\alpha}\right\}_{\alpha}^{n},\left\{v_{\alpha}\right\}_{\alpha}^{n},\left\{A_{\alpha}^{\beta \gamma}\right\}_{\alpha \beta \gamma}^{n}\right)=\frac{\mathbf{v} \cdot \overline{\mathcal{A}}_{k}^{-1} \cdot \mathbf{v}}{\mathbf{v} \cdot \overline{\mathcal{A}}_{k}^{-1} \cdot \mathbf{v}_{k}},
$$

where

$$
\begin{aligned}
& \mathbf{v}=\left\{v_{\alpha}\right\}_{\alpha}^{n}, \quad \overline{\mathbf{v}}_{k}=\left\{v_{\alpha} k_{\alpha}^{-1}\right\}_{\alpha}^{n}, \\
& \overline{\mathcal{A}}_{k}=\left\{\frac{4}{9} v_{\alpha} k_{\alpha}^{-1} \delta_{\alpha \beta}+\sum_{\gamma=1}^{n} A_{\gamma}^{\alpha \beta}\left(2 \mu_{\gamma}\right)^{-1}\right\}_{\alpha \beta}^{n} .
\end{aligned}
$$

The trial field (4), (22) containing free parameters $a_{\alpha}(\alpha=1, \ldots, n)$ restricted by (6), are more general than the Hashin - Shtrikman ones used in $[2,4]$, hence the bounds (19), (24) obtained here are more restricted. Applications of the bounds will be given in the next section. 


\section{APPLICATIONS}

In the case of two-component materials, the three-point correlation parameters $A_{\alpha}^{\beta \gamma}$ can be expressed through just one independent parameter [7]

$$
\begin{aligned}
& A_{\alpha}^{11}=A_{\alpha}^{22}=-A_{\alpha}^{12}=\frac{2}{3} v_{1} v_{2} \zeta_{\alpha}, \quad \alpha=1,2 \\
& \zeta_{1}+\zeta_{2}=1, \quad 0 \leq \zeta_{1}, \zeta_{2} \leq 1
\end{aligned}
$$

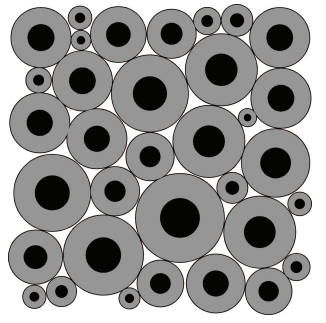

(a)

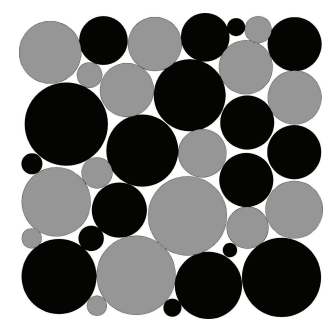

(b)

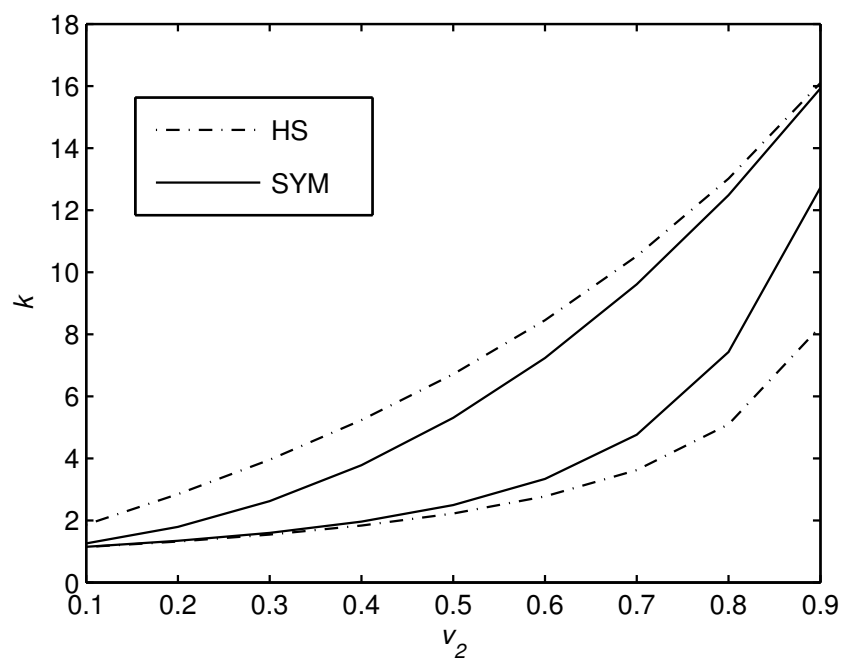

(c)

Fig. 1. Bounds on the elastic bulk moduli of two-phase coated spheres and symmetric spherical cell mixture at $k_{1}=1, \mu_{1}=0.3, k_{2}=20, \mu_{2}=10, v_{2}=0.1 \rightarrow 0.9$. (a) Coated spheres; (b) Symmetric spherical cell mixture; (c) HS - HashinStrikman upper and lower bounds and also the respective exact effective bulk moduli of the coated spheres at $\zeta_{2}=1$ and $\zeta_{1}=0$, SYM - upper and lower bounds for the symmetrical spherical cell mixtures.

For Hashin-Shtrikman poly-dispersed two-phase coated sphere model where the coated sphere with the same volume proportions of phases fill all the material space 
(Fig. 1a), the parameters $\zeta_{\alpha}$ have been determined exactly and analytically [7, 8]. In particular $\zeta_{2}=1$ if phase-2 is the matrix phase, and $\zeta_{2}=0$ if it is the inclusion phase. Moreover the upper (19) and lower (24) bounds converge to the unique effective bulk modulus, which coincides with Hashin- Shtrikman upper (lower) bound if the phase-2 is the matrix (inclusion) phase, provided that $\mu_{2}>\mu_{1}$. Fig. 1c presented the exact effective bulk moduli for those alternative coated spheres, which are also Hashin-Shtrikman upper and lower bounds for two-component materials, in the range $v_{2}=0.1 \rightarrow 0.9$, with $k_{1}=1, \mu_{1}=0.3, k_{2}=20, \mu_{2}=10$ (here and afterward $k_{1}$ is normalized to be unity).

For two-phase symmetric spherical cell mixtures (Fig. 1b) without distinct inclusion and matrix phases, we have $\zeta_{\alpha}=v_{\alpha}[6,7]$. The upper and lower bounds for those mixtures are projected in Fig. 1c. Though the bounds do not converge in the case, they fall inside the Hashin-Shtrikman bounds.

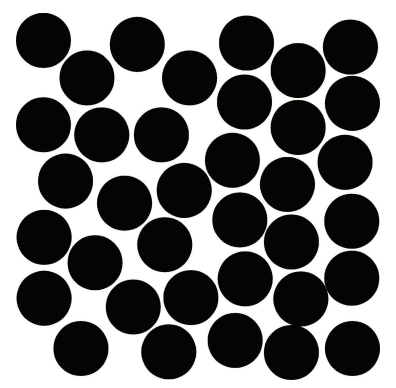

(a)

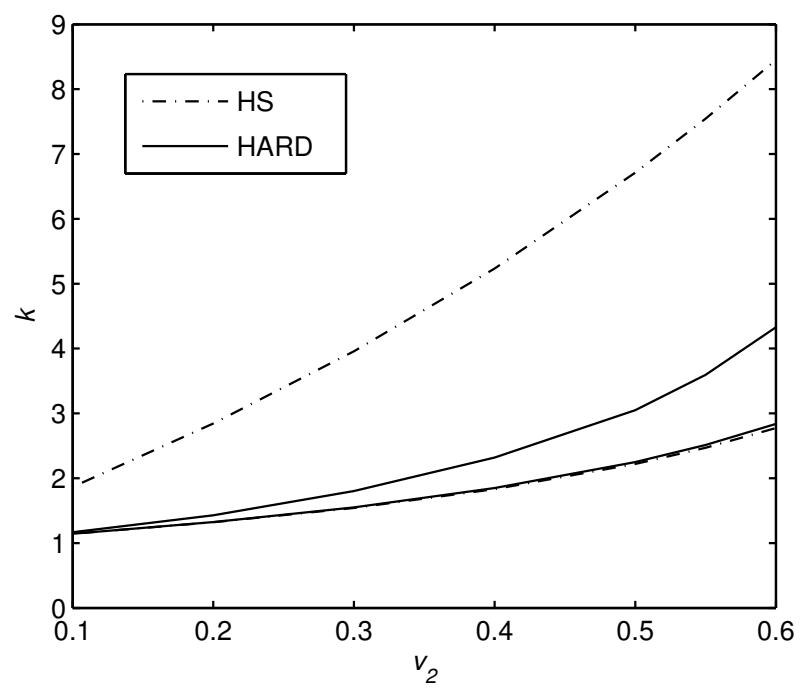

(b)

Fig. 2. Hashin-Strikman bounds (HS) and the bounds (HARD) on the elastic bulk modulus of the random suspension of equisized hard spheres. (a) A random suspension of equisized hard spheres ; (b) The bounds, $k_{1}=1, \mu_{1}=0.3, k_{2}=$ $20, \mu_{2}=10, v_{2}=0.1 \rightarrow 0.6$.

Now consider the two-phase random suspensions of equisized hard spheres (Fig. 2a) and overlapping spheres (Fig. 3a) in a base phase-1. The bounds (19) and (24) for the models at range $v_{2}=0 \rightarrow 0.99$, with $k_{1}=1, \mu_{1}=0.3, k_{2}=20, \mu_{2}=10$, together with Hashin-Shtrikman bounds are projected in Figs. 2a, 3b. The three-point correlation parameter $\zeta_{2}$ for the models has been calculated and tabulated in [6] and given in Table 1.

Now we come to the three-phase doubly-coated sphere model (Fig. 4a), where the composite spheres of all possible sizes but with the same volume proportions of phases fill 
Table 1. Three-point correlation parameter $\zeta_{2}$ for two-phase random suspensions of hard spheres and overlapping spheres in a base phase-1

\begin{tabular}{|c|c|c|}
\hline \multirow{2}{*}{$v_{2}$} & \multicolumn{2}{|c|}{ Three-point parameter $\zeta_{2}$} \\
\cline { 2 - 3 } & Hard spheres & Overlaping spheres \\
\hline 0.00 & 0.000 & 0.000 \\
0.10 & 0.020 & 0.056 \\
0.20 & 0.041 & 0.114 \\
0.30 & 0.060 & 0.171 \\
0.40 & 0.077 & 0.230 \\
0.50 & 0.094 & 0.290 \\
0.55 & 0.110 & 0.320 \\
0.60 & 0.134 & 0.351 \\
0.70 & - & 0.415 \\
0.80 & - & 0.483 \\
0.90 & - & 0.558 \\
0.95 & - & 0.604 \\
0.99 & - & 0.658 \\
\hline
\end{tabular}



(a)

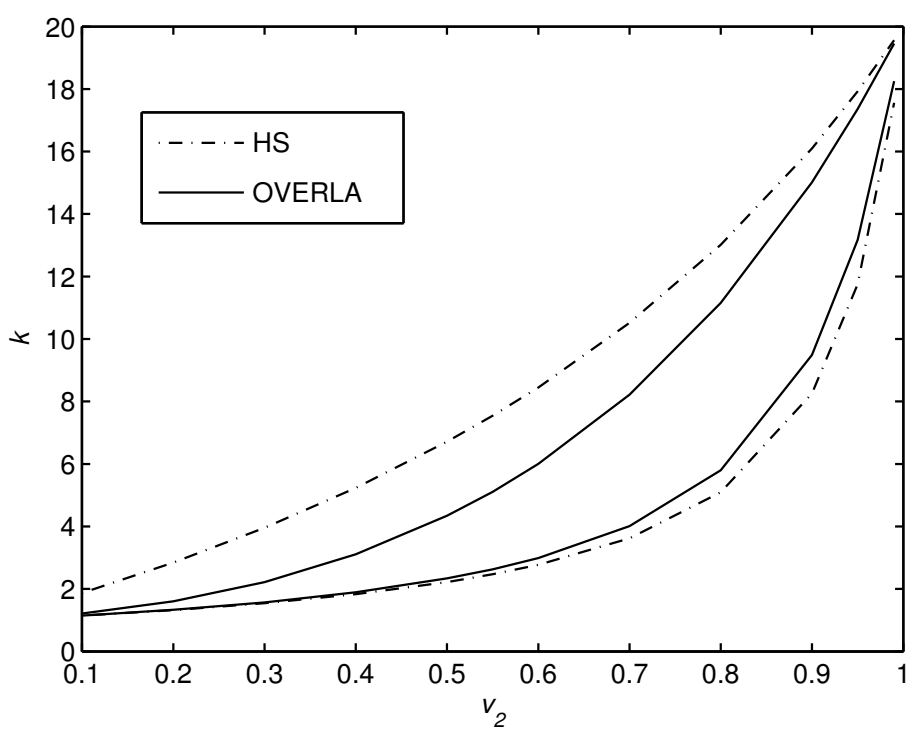

(b)

Fig. 3. Hashin-Strikman bounds (HS) and the bounds (OVERLA) on the elastic bulk modulus of the random suspension of equisized overlaping spheres. (a) A random suspension of equisized overlaping spheres ; (b) The bounds, $k_{1}=1, \mu_{1}=$ $0.3, k_{2}=20, \mu_{2}=10, v_{2}=0.1 \rightarrow 0.99$. 
all the material space - an extension of Hashin-Shtrikman two-phase model. The three-

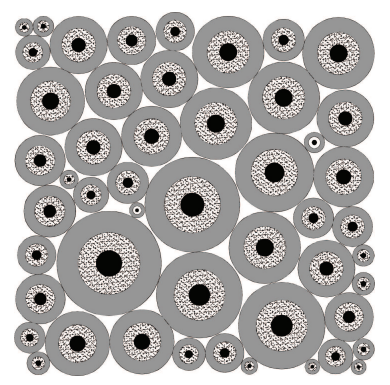

(a)



(b)

Fig. 4. Bounds on the elastic bulk modulus of doubly coated spheres, $k_{1}=$ $12, \mu_{1}=8, k_{2}=1, \mu_{2}=0.3, k_{3}=30, \mu_{3}=15, v_{1}=0.1 \rightarrow 0.9, v_{2}=v_{3}=\frac{1}{2}\left(1-v_{1}\right)$. (a) Doubly coated spheres ; (b) Hashin-Strikman (HS), old bounds and the new bounds which converges to the exact value of the effective modulus.

point correlation parameters $A_{\alpha}^{\beta \gamma}$ of this three-phase model also have been determined analytically in [8]

$$
\begin{aligned}
& A_{1}^{11}=A_{1}^{12}=A_{1}^{13}=A_{1}^{21}=A_{1}^{22}=A_{1}^{23}= \\
&=A_{1}^{31}=A_{1}^{32}=A_{1}^{33}=A_{2}^{13}=A_{2}^{31}=A_{2}^{23}=A_{2}^{32}=A_{2}^{33}=0, \\
& A_{2}^{11}=A_{2}^{22}=-A_{2}^{12}=-A_{2}^{21}=\frac{2}{3} \frac{v_{1} v_{2}}{\left(v_{1}+v_{2}\right)}, \quad A_{3}^{11}=\frac{2}{3} \frac{v_{1}^{2} v_{3}}{\left(v_{1}+v_{2}\right)}, \\
& A_{3}^{12}=A_{3}^{21}=\frac{2}{3} \frac{v_{1} v_{2} v_{3}}{\left(v_{1}+v_{2}\right)}, \quad A_{3}^{13}=A_{3}^{31}=-\frac{2}{3} v_{1} v_{3}, \quad A_{3}^{22}=\frac{2}{3} \frac{v_{2}^{2} v_{3}}{\left(v_{1}+v_{2}\right)}, \\
& A_{3}^{23}=A_{3}^{32}=-\frac{2}{3} v_{2} v_{3}, \quad A_{3}^{33}=\frac{2}{3} v_{3}\left(v_{1}+v_{2}\right) .
\end{aligned}
$$

The bounds (12) and (24) also converge to the exact effective bulk modulus of the model, see Fig. 4b. In the figure Hashin - Shtrikman bounds [1] and the old correlation bounds in [2] are also presented for comparisons, at the range $v_{1}=0.1 \rightarrow 0.9, v_{2}=v_{3}=$ $\frac{1}{2}\left(1-v_{1}\right)$ with $k_{1}=12, \mu_{1}=8, k_{2}=1, \mu_{2}=0.3, k_{3}=30, \mu_{3}=15$ (the inner sphere of phase 3 is embedded in the shell made of phase 2 , and then the coated sphere is embedded in the shell of phase-1). 
Lastly we come to the symmetric cell material (Fig. 5a) without distinct inclusion and matrix phase [3,6]. For the material we have [3] $(\alpha \neq \beta \neq \gamma \neq \alpha)$

$$
\begin{aligned}
& A_{\alpha}^{\beta \gamma}=v_{\alpha} v_{\beta} v_{\gamma}\left(f_{1}-f_{3}\right), \quad A_{\alpha}^{\alpha \alpha}=v_{\alpha}\left(1-v_{\alpha}\right)\left[\left(1-v_{\alpha}\right) f_{1}+v_{\alpha} f_{3}\right] \\
& A_{\alpha}^{\alpha \beta}=v_{\alpha} v_{\beta}\left[\left(v_{\alpha}-1\right) f_{1}-v_{\alpha} f_{3}\right], \quad A_{\alpha}^{\beta \beta}=v_{\alpha} v \beta\left[\left(1-v_{\beta}\right) f_{3}+v_{\beta} f_{1}\right]
\end{aligned}
$$

which depend on just 2 shape parameters $f_{1}, f_{3}$ subjected to the limitations

$$
f_{1}+f_{3}=\frac{2}{3}, \quad 0 \leq f_{1}, f_{3} \leq \frac{2}{3} .
$$

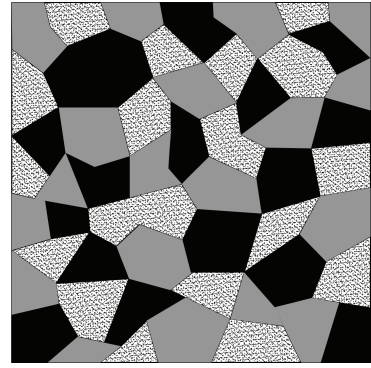

(a)



(b)

Fig. 5. Hashin-Strikman bounds (HS) and bounds (SYM) on the elastic bulk modulus of three-phase symmetric cell mixtures, $k_{1}=1, \mu_{1}=0.3, k_{2}=12, \mu_{2}=$ $8, k_{3}=30, \mu_{3}=15, v_{1}=0.1 \rightarrow 0.9, v_{2}=v_{3}=\frac{1}{2}\left(1-v_{1}\right)$. (a) A symmetric cell mixture; (b) The bounds.

The bounds on the effective bulk modulus of symmetric cell materials become

$$
K_{S Y M}^{U}\left(\left\{k_{\alpha}\right\}_{\alpha}^{n},\left\{\mu_{\alpha}\right\}_{\alpha}^{n},\left\{v_{\alpha}\right\}_{\alpha}^{n}\right) \geq k^{e f f} \geq K_{S Y M}^{L}\left(\left\{k_{\alpha}\right\}_{\alpha}^{n},\left\{\mu_{\alpha}\right\}_{\alpha}^{n},\left\{v_{\alpha}\right\}_{\alpha}^{n}\right),
$$

where

$$
\begin{aligned}
& K_{S Y M}^{U}\left(\left\{k_{\alpha}\right\}_{\alpha}^{n},\left\{\mu_{\alpha}\right\}_{\alpha}^{n},\left\{v_{\alpha}\right\}_{\alpha}^{n}\right)=\max _{0 \leq f_{1} \leq \frac{2}{3}} K_{A}^{U}\left(\left\{k_{\alpha}\right\}_{\alpha}^{n},\left\{\mu_{\alpha}\right\}_{\alpha}^{n},\left\{v_{\alpha}\right\}_{\alpha}^{n},\left\{A_{\alpha}^{\beta \gamma}\right\}_{\alpha \beta \gamma}^{n} \in(28),\right. \\
& K_{S Y M}^{L}\left(\left\{k_{\alpha}\right\}_{\alpha}^{n},\left\{\mu_{\alpha}\right\}_{\alpha}^{n},\left\{v_{\alpha}\right\}_{\alpha}^{n}\right)=\min _{0 \leq f_{1} \leq \frac{2}{3}} K_{A}^{L}\left(\left\{k_{\alpha}\right\}_{\alpha}^{n},\left\{\mu_{\alpha}\right\}_{\alpha}^{n},\left\{v_{\alpha}\right\}_{\alpha}^{n},\left\{A_{\alpha}^{\beta \gamma}\right\}_{\alpha \beta \gamma}^{n} \in(28),\right.
\end{aligned}
$$


Numerical result for the bounds on the effective bulk modulus of three-phase symmetric cell materials, at the range $v_{1}=0.1 \rightarrow 0.9, v_{2}=v_{3}=\frac{1}{2}\left(1-v_{1}\right)$ with $k_{1}=1, \mu_{1}=$ $0.3, k_{2}=12, \mu_{2}=8, k_{3}=30, \mu_{3}=15$, are presented in Fig. 5 b, with fall inside HashinShtrikman bounds for the large class of isotropic composites. The result are also reported in Tab. 2, with the values of $f_{1}$ where the respective Max and Min in (31) are reached.

Table 2. Hashin-Shtrikman bounds $\left(k_{H S}^{U}, k_{S H}^{L}\right)$ and the bounds for symmetric cell three-phase mixture $\left(k_{S Y M}^{U}, k_{S Y M}^{L}\right)$ at $v_{1}=0.1 \rightarrow 0.9, v_{2}=v_{3}=\frac{1}{2}\left(1-v_{1}\right)$ with $k_{1}=1, \mu_{1}=0.3, k_{2}=12, \mu_{2}=8, k_{3}=30, \mu_{3}=15 ; f_{1}^{\text {max }}$ and $f_{1}^{\text {min }}$ the values where the Max and Min in (31) are reached

\begin{tabular}{|c|c|c|c|c|c|c|}
\hline$v_{1}$ & $k_{S H}^{U}$ & $k_{S Y M}^{U}$ & $k_{S Y M}^{L}$ & $k_{S H}^{L}$ & $f_{1}^{\max }$ & $f_{1}^{\min }$ \\
\hline 0.1 & 15.9397 & 15.2146 & 8.1666 & 7.7618 & 0 & $2 / 3$ \\
0.2 & 13.3069 & 12.1270 & 5.2193 & 4.9114 & 0 & $2 / 3$ \\
0.3 & 11.0335 & 9.4753 & 3.8304 & 3.5366 & 0 & $2 / 3$ \\
0.4 & 9.0507 & 7.2301 & 3.0183 & 2.7272 & 0 & 0.6391 \\
0.5 & 7.3060 & 5.6505 & 2.4590 & 2.1938 & $2 / 3$ & 0 \\
0.6 & 5.7590 & 4.6712 & 1.9429 & 1.8159 & $2 / 3$ & 0 \\
0.7 & 4.3779 & 3.7264 & 1.5894 & 1.5341 & $2 / 3$ & 0 \\
0.8 & 3.1373 & 2.8038 & 1.3355 & 1.3159 & $2 / 3$ & 0 \\
0.9 & 2.0169 & 1.8965 & 1.1459 & 1.1419 & $2 / 3$ & 0 \\
\hline
\end{tabular}

\section{CONCLUSIONS}

In this paper new third order three-point correlation bounds on the elastic bulk modulus of $n$ component materials have been constructed, which coincide with our previous bounds in the two-component material case, but are tighter for $n \geq 3$. It is interesting that the bounds converge to the unique effective bulk modulus of multi - coated spheres (extension of Hashin - Shtrikman two - component one) - the unique case where the three - point correlation parameters have been determined analytically. These bounds are also specified for symmetric cell materials. Some examples involving numerical values of three-point correlation parameters for certain particular micro - geometries taken from literature, are also presented for more illustrations.

\section{ACKNOWLEDGEMENT}

The authors thank the financial support of Vietnam's Nafosted.

\section{REFERENCES}

[1] Hashin Z., Shtrikman S., A variational approach to the theory of the elastic behaviour of multiphase materials, J.Mech.Phys. Solids 11 (1963) 127-140.

[2] Pham D. C., Bounds on the effective shear modulus of multiphase materials, Int.J, Engng. Sci 31 (1993) 11-17. 
[3] Pham D. C., Bounds for the effective conductivity and elastic moduli of fully-disordered multicomponent materials, Arch. Rational Mech. Anal 127 (1994) 191-198.

[4] Pham D. C.,Bounds for the effective properties of isotropic composite and poly-crystals, D. Sci. Thesis, Hanoi (1996).

[5] Milton G. W., The theory of Composites, Cambridge University Press (2001).

[6] Torquato S., Random heterogeneous media, New York, Springer (2002).

[7] Pham D.C., Torquato S., Strong-contrast expansions and approximations for the effective conductivity of isotropic multiphase composites, J. Appl, Phys, 94 (2003) 6591-6602.

[8] Pham D.C., Estimations for the overall properties of some isotropic locally-ordered composites, Acta, Mech, 121 (1997) 177-190.

Received November 5, 2011 